\title{
11 Psychosoziale Faktoren des Alterns
}

\author{
Kurt Seikowski
}

\subsection{Auch Männer werden älter - Älterwerden und Gesundheit}

Die Lebenserwartung hat sich in der Vergangenheit kontinuierlich im Allgemeinen infolge des medizinischen Fortschritts erhöht. Eine weitere Entwicklung ist die Verringerung der Differenz in der Lebenserwartung zwischen Männern und Frauen. Für das Jahr 206o ergibt sich für Männer eine durchschnittliche Lebenserwartung bei Geburt von 85,0 Jahren und für Frauen von 89,2 Jahre (Statistisches Bundesamt 2009). Das bedeutet aktuell, dass 65-jährige Männer immer noch mit weiteren 22,3 beziehungsweise Frauen mit 25,5 Jahren rechnen können. Das sind jeweils rund 5 Jahre mehr als 2006/2008. Dies bedeutet mit großer Wahrscheinlichkeit eine stetig steigende Lebenserwartung der deutschen Männer bei immer besserer Gesundheit und steigendem Verlangen nach hoher Lebensqualität insbesondere bei typisch maskulinen Bedürfnissen.

Somatische und psychosoziale Faktoren des Alterns beim Mann werden in den letzten Jahren unter dem Begriff der „alternde Mann“ („aging male“) zusammengefasst. Dieser Begriff umfasst im eigentlichen Sinne zwei Entwicklungsabschnitte:

- das mittlere Lebensalter von ca. 40 bis 65 Jahren sowie

- das späte Lebensalter, das mit dem Eintritt in das Rentenalter ab 65 Jahren auch als Seniorenalter bezeichnet wird (Brandstädter $u$. Lindenberger 2007).

Es gibt viele Gründe, sich mit diesen zwei Lebensabschnitten zu beschäftigen. Sind es doch Phasen in der Entwicklung von Männern, wo das bisherige a priori von vielen Männern implizite „perfekte Männerbild“ nicht mehr funktioniert, was auch als „männlicher Geschlechtsrollenstress“ bezeichnet wird (Daig 2009). Auch historisch gesehen hatte der Mann immer perfekt zu sein - und es wurden dem Mann kaum Möglichkeiten „gegönnt“, auch mal schwach zu sein. Schon immer galten Männer als das „starke“ Geschlecht gegenüber den Frauen, dem „schwachen“ Geschlecht (Dinges 2007a und b). Dabei fällt auch auf, dass die medizinische Forschung dieses „Vorurteil“ auch noch unterstützt, indem sie den Männern (den Frauen auch) über 
die Floskel „Anti-Aging“ das Gefühl vermittelt, ewig jung bleiben zu können. Dahinter steht natürlich auch die Angst, dass sich mit zunehmendem Alter auch die Cesundheit verschlechtert. Dabei ist dem nicht so, denn neben dem pathopsychologischen und pathophysiologischen Konzept des Alterns werden zunehmend auch positive Aspekte (salutogenetisches Konzept) mit dem Älterwerden assoziiert (s. Tab. 1).

\section{Tab. 1 Psychologische Konzepte des Alterns}

pathopsychologisches und pathophysiologisches Konzept

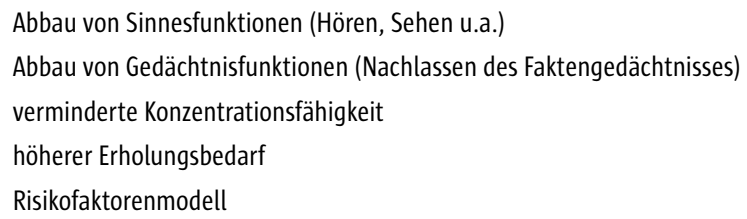

Ab einem bestimmten Alter kann es durchaus sein, dass zunächst eine Lesebrille benötigt wird, Fakten schneller als vorher vergessen werden, die Konzentrationsfähigkeit nachlässt, mehr Erholung für die eigene Regenerierung erforderlich ist und bestehende Risikofaktoren wie Rauchen, Alkohol, Adipositas u.a. zunehmend chronische Krankheiten nach sich ziehen. Doch gleichermaßen kann man aufgrund von Lebenserfahrung Krisen besser meistern. Oft hat sich auch ein sehr guter unterstützender Freundes- und Bekanntenkreis etabliert. Das abstrakte Denken und auch das Zusammenhangswissen als Gedächtnisleistungen nehmen sogar noch zu. Berufliche und familiäre Sicherheiten stellen zunehmend auch schützende Faktoren für die eigene Gesundheit dar.

Aber natürlich bleibt der Umstand bestehen, dass mit zunehmendem Alter nicht nur für die Frau sondern auch für den Mann altersbedingt das Risiko von Krankheiten zunimmt. Doch wenngleich für viele Frauen Vorsorgeuntersuchungen und der Gang zum Arzt bei auftretenden Beschwerden schon selbstverständlich sind, haben Männer dabei einige Probleme. Durch das historisch gewachsene „perfekte“ Männerkonzept werden sie nach Dinges (2009) zu „beratungsresistenten Gesundheitsidioten“. Das betrifft u.a. alle männerspezifischen somatoformen Störungen wie z.B. unspezifisches Ziehen im Genitalbereich, hypochondrische Störungen in Bezug auf männliche Körpermerkmale wie den Penis (Harth et al. 2007) und Männererkrankungen wie Prostatakrebs oder auch Formen der Prostatitis wie auch Beschwerden im Vorfeld von Hypertonie, Herzinfarkten und Schlaganfällen (Seikowski 2008). Sie nehmen Beratungsangebote weniger oft in Anspruch, gehen seltener zum Arzt, interessieren sich weniger für ihre Gesundheit als Frauen. 
Durch die Anti-Aging-Debatte wird den Männern zusätzlich suggeriert, dass sie keine Angst vor dem Altern haben müssen, denn die sich mittlerweile so nennende „Lifestyle-Medizin“ zeigt ihnen - oft in Form von Medikamenten (z.B. Viagra, Levitra und Cialis bei Potenzproblemen oder Propecia bei Haarausfall) - dass man seinen bisherigen Lebensstil so weiterleben könne wie bisher (Harth et al. 2008).

Die nachfolgenden Untersuchungen belegen jedoch, dass dem nicht so ist.

Es gibt auch neue Probleme im Zusammenhang mit dem zunehmenden Alter bei Männern, die ebenfalls nicht außer Acht gelassen werden sollten, denn auch das Reproduktionsalter von Erstvätern nimmt immer mehr zu. Das bedeutet, dass das Bekommen von Kindern nicht mehr nur eine Problematik von jungen Männern darstellt. Altern und das Zeugen von Nachwuchs widersprechen sich nicht und werden immer mehr zur gesellschaftlichen Realität.

Ebenso verändert sich der Mann mit zunehmendem Alter auch hormonell. Was diesbezüglich bei Frauen schon lange in der Wissenschaft thematisiert wurde, wird in den letzten Jahren auch zunehmend bei Männern beforscht (s. Kap. II.13).

\subsection{Entwicklungspsychologische Besonderheiten des älter werdenden Mannes}

Mittlerweile existieren sehr umfangreiche Studien und Lehrbücher, die sich mit dem Alter und dem Älterwerden auseinandersetzen - aus sozialwissenschaftlicher (Backes u. Clemens 2008), entwicklungspsychologischer (Brandstätter u. Lindenberger 2007) sowie interdisziplinärer gerontologischer Sicht (Baltes et al. 1994). Obwohl dort gesundheitsspezifische Aspekte von Männern nicht per se im Vordergrund stehen, sind sie für zukünftige Studien von hohem heuristischen Wert, da in ihnen konzeptionelle Grundlagen dargestellt sind, die auch für die Männergesundheit von Bedeutung sind.

So unterscheiden z.B. Gerock und Brandstädter (1994) normales, krankhaftes und optimales Altern.

- Unter „normalem Altern“ verstehen sie das Erreichen einer durchschnittlichen Lebensspanne, die lediglich durch alterstypische Einbußen auf der psychischen und der organisch-somatischen Ebene beeinträchtigt ist.

- Beim „krankhaften Altern“ ist der Alterungsprozess durch das Auftreten von spezifischen Krankheitssymptomen. Leistungs- und Funktionseinschränkungen gekennzeichnet. Häufig kommt es dabei zu einer verkürzten Lebensspanne bei gleichzeitig eingeschränkter Lebensqualität.

- Beim „optimalen Altern“ wird der Alterungsprozess so charakterisiert, dass die erreichte Lebenszeit, die organische Funktionstüchtigkeit sowie auch die subjektive Lebensqualität gegenüber vergleichbaren Populationen deutlich erhöht sind. Gemeint sind damit vor allem Personen, die sich aktiv mit dem Alter auseinandersetzen.

Was jedoch verändert sich mit zunehmendem Alter? Die in den Tabellen 2 und 3 dargestellten Veränderungen für das mittlere und das späte Lebensalter werden oft als kritische Lebensereignisse (Filipp 2007) erlebt. Dabei geht es um die Frage,

- welches Lebensereignis wirkt bei

- welchen Personen mit

- welchen Eigenschaften 
- zu welchem Zeitpunkt

- unter Einwirkung welcher Faktoren

- in welcher Weise, d.h. ruft Störungen welcher Art hervor, und

- welche Wirkmechanismen spielen dabei eine Rolle (Richter u. Guthke 1996).

Das Ergebnis des Erlebens derartiger Lebensereignisse ist immer eine Neuanpassung oder eine Verleugnung. Beide Prozesse sind aber auch im Rahmen von Verarbeitungsprozessen spürbar - körperlich (z.B. Magenbeschwerden) wie psychisch (Ängste, Depressionen). Die dazugehörige Datenlage für Männer im deutschsprachigen Raum soll dazu im Folgenden dargestellt werden.

\section{Tab. 2 Entwicklungspsychologische Besonderheiten im mittleren Lebensalter}

Wahrnehmung körperlicher Veränderungen

Wahrnehmung einer eingeschränkten Leistungsfähigkeit

Beeinträchtigung des bisherigen „perfekten“ Männerkonzepts

Kinder sind aus dem Haus

neue direkte Formen der Wahrnehmung der eigenen Partnerschaft

erhöhtes Trennungsrisiko und mögliche neue Beziehungsaufnahme mit einer jüngeren Frau mit Kinderwunsch

Stagnation der beruflichen Entwicklung

meist erstmalige Konfrontation mit chronischen Erkrankungen

meist erstmalige Konfrontation mit dem Tod eigener Eltern

meist erstmalige Konfrontation mit dem Tod Gleichaltriger

meist erstmalige Konfrontation mit der neuen Rolle als „0pa“

meist erste sexuelle Beeinträchtigungen (weniger Spontanerektionen, Libidomangel u.a.)

Einschränkungen im Genussbereich (Alkohol wird nicht mehr so vertagen wie früher u.a.)

\section{Tab. 3 Entwicklungspsychologische Besonderheiten im späten Lebensalter}

Umstellung auf das Rentenalter

erneute Änderung der Partnerschaftssituation durch „Ganztagskontakt“

Neuanpassung hinsichtlich täglicher Aufgaben und Verpflichtungen

mehr Zeit für Hobbys und andere Aktivitäten

zunehmende Beschäftigung mit Krankheiten und altersbedingten Veränderungen des Körpers

Krankheit und Tod der meist langjährigen Partnerin bzw. des meist langjährigen Partners

zunehmende Konfrontation mit Krankheiten und dem Tod von Freunden und Bekannten

Altersdepression

Konfrontation mit der Möglichkeit von altersgerechtem Wohnen bis hin zum Pflegeheim

\subsubsection{Subjektive körperliche und psychische Beschwerden des alternden Mannes}

An 2.144 Männern aus Deutschland wurden in einer Querschnittsstudie mit standardisierten Fragebögen (Fragebogen zur Lebenszufriedenheit FLZ, Generalized Anxiety 
Disorder GAD-7, Gesundheitsfragebogen für Patienten PHQ-2, Fragebogen zur Erfassung der psychischen Widerstandsfähigkeit RS-11, Rosenbergskala zum globalen Selbstwertgefühl RSS) Veränderungen hinsichtlich der Merkmale Lebenszufriedenheit, Angst, Depression und Resilienz untersucht (Beutel et al. 2010). Die Untergliederung der zu vergleichenden Gruppen erfolgte in Dekaden (von 18-30, bzw. 31-40 bis hin $z u>70$ ). Hinsichtlich der Lebenszufriedenheit gab es keine altersabhängigen Veränderungen. Die Zufriedenheit mit der Gesundheit verringerte sich bei den Männern zwischen 51-6o. Gleichzeitig stieg in dieser Gruppe das Gesundheitsbewusstsein an. Auch waren in dieser Altersgruppe die Angstwerte erhöht sowie Resilienz und Selbstwert erniedrigt. Hinsichtlich der Depressionswerte gab es keine klaren Aussagen. Die Zufriedenheit mit der Partnerschaft und der Sexualität nahm nur bei den über 7o-Jährigen ab. Eine höhere Lebenszufriedenheit korrelierte mit höheren Werten in den Merkmalen Resilienz, gegebener Arbeitstätigkeit, dem Vorhandensein einer Partnerschaft sowie mit höherem Selbstwert und einem guten finanziellen Einkommen. Insgesamt zeigte sich also eine psychische Destabilisierung bei den 51- bis Go-Jährigen.

Aber offenbar sind derartige Ergebnisse auch fragebogenabhängig, denn in früheren Untersuchungen (zusammengestellt bei Merbach et al. 2007) mit dem HADS (Hospital Anxiety and Depression Scale) zur Messung von Angst von Depressionen stiegen die Depressionswerte mit zunehmendem Alter an (siehe auch Glaesmer et al. 2010).

Eine weitere Studie hatte die „Midlife-Crisis“ als Gegenstand (Seikowski et al. 2007). Zur Untersuchung dieser Problematik wurden anonym in brieflicher Form 85 Männer zwischen 40 und 65 Jahren befragt. Sie füllten 2 Fragebögen aus: das Klimakterium-Virile-Beschwerden-Inventar (KLV) und den Fragebogen zur Lebenszufriedenheit (FLZ). Es erfolgte eine Unterteilung dieser Personen mittels Prozessanalyse in 3 Gruppen: $40-47(\mathrm{~N}=26), 48-55(\mathrm{~N}=33)$ und 56-65 $(\mathrm{N}=26)$ Jahre. Auffällig in diesen Personengruppen war ein sehr hoher Anteil von Personen mit Hochschulabschluss. Die häufigsten psychischen Veränderungen betrafen Konzentrationsstörungen $(72,9 \%)$, Müdigkeit $(70,6 \%)$, gesteigerte Reizbarkeit $(65,9 \%)$ und Nachlassen des Gedächtnisses $(63,5 \%)$. Bei den körperlichen Veränderungen wurden am häufigsten Gelenk- und Gliederschmerzen (77,6\%) genannt, gefolgt von Kreuzschmerzen (68,2\%), dem Nachlassen des Interesses an Sexualität $(62,6 \%)$ und einer Gewichtszunahme $(55,0 \%)$. Während sich die 40 bis 47-Jährigen in vielen Merkmalen nicht von den 56bis 65-Jährigen unterschieden, war die Lebenszufriedenheit in der mittleren Gruppe der 48- bis 55-Jährigen hinsichtlich der Merkmale Freizeit, Partnerschaft, eigene Sexualität, Zufriedenheit mit sich selbst sowie soziale Integration verringert. Das bedeutet, dass es im Alter um die 50 Jahre zu einer Destabilisierung in vielen Lebensbereichen kommt. Nach dieser Periode sind die Männer jedoch in der Lage, sich an ihre veränderten körperlichen und psychischen Veränderungen auf einem offenbar qualitativ anderen Niveau als früher neu anzupassen. Was diese Veränderungen bewirkte, konnte mit dieser Studie jedoch noch nicht erfasst werden.

Merbach et al. (2007) fanden unter Verwendung des Giessener Beschwerdebogens (GBB) hinsichtlich der Herz-, Magen- und Gelenkbeschwerden, des Beschwerdendrucks, hinsichtlich Angst und Depression eine positive Korrelation mit zunehmendem Alter.

Thiele (1999) untersuchte 304 gesunde Männer zwischen 35 und 64 Jahren hinsichtlich des Zusammenhanges zwischen körperlichem Altern und psychischem Befin- 
den. Dabei konnte gezeigt werden, dass trotz der Abnahme der körperlichen Leistungsfähigkeit mit zunehmendem Alter sich die Männer subjektiv dadurch nicht beeinträchtigt fühlen. Damit konnte allerdings nicht geklärt werden, ob die Männer die Abnahme der körperlichen Leistungsfähigkeit als normal erleben, sich deshalb subjektiv nicht beeinträchtigt fühlten oder aber Verdrängungsprozesse bedeutsam waren.

In einer ähnlich konzipierten Studie bei Männern ( $\mathrm{N}=383)$ und Frauen ( $\mathrm{N}=405)$ wurde clusteranalytisch der Zusammenhang zwischen Körperbild und körperlichem Wohlbefinden im Alter für 4 Altersgruppen ab 64 Jahren untersucht. Trotz der körperlichen Alterungsprozesse wird der Körper bei den Männern (und Frauen) nicht negativer wahrgenommen. Körperbezogene Aktivitäten gehen allerdings auch mit zunehmendem Alter zurück, sodass der Körper auch weniger belastet wird (Albani et al. 2009). Offenbar stellen sich älter werdende Personen auch mehr auf ihre körperlichen Einschränkungen ein, wodurch die Einschränkungen auch nicht als zusätzlich belastbar erlebt werden.

Hinsichtlich der Männerdepressionen spielt der Suizid bei erfolgreichen Männern noch eine gesonderte Rolle. Die Tendenz, dass sich Männer 2- bis 3-mal häufiger suizidieren als Frauen - und es sich dabei auch vermehrt um ältere Männer handelt, ist seit über 150 Jahren konstant. Als Gründe vermutet Wolfersdorf (2009) für die erfolgreichen Männer vor allem Perfektionismus und Scham. Allerdings ist die Suizidrate bei Männern von 1990 zu 2007 auch leicht gesunken von 24,9 auf 17,4 Personen auf 100.00o Einwohner und Jahr.

\subsubsection{Altern ab 70 - Die Berliner Altersstudie (BASE)}

Eine besonders umfangreiche Untersuchung bei der Erforschung des Alterns von Männern (und Frauen) stellt die Berliner Altersstudie dar (Lindenberger et al. 2010). Zu mehreren Zeitpunkten wurden in der Westberliner Bevölkerung (beginnend mit 1.908 Personen) Erhebungen zu den vielfältigsten Lebensbereichen durchgeführt. Erfasst wurden Personen, die 7o Jahre und älter waren. Für Männer und Frauen gleichermaßen konnten folgende wichtige Daten zum Altern erhoben werden, wobei sich Männer und Frauen in den verschiedenen Bereichen kaum voneinander unterschieden:

- Die meisten alten Menschen erhalten regelmäßig ärztlich verordnete Medikamente. $92 \%$ werden mit mindestens einem, $24 \%$ mit fünf und mehr Medikamenten behandelt.

- Bei nahezu allen Personen ließ sich mindestens eine Krankheit nach den ICDKriterien diagnostizieren.

v Allerdings beurteilen 29\% dieser Personen ihre körperliche Gesundheit als gut bis sehr gut, $38 \%$ als befriedigend, $33 \%$ als ausreichend und $14 \%$ als mangelhaft.

- Obwohl Frauen eine höhere Lebenserwartung haben, unterscheiden sie sich nicht in ihrer körperlich-organischen Gesundheit von gleichaltrigen Männern. Männer leiden sogar unter weniger Erkrankungen als Frauen.

- Depressive Erkrankungen nehmen in der untersuchten Stichprobe ab 70 nicht signifikant zu. 
- Nur ca. $17 \%$ leiden an pathologischen kognitiven Störungen -14\% hatten davon das Ausmaß einer Demenz. Die Demenzhäufigkeit steigt jedoch mit zunehmendem Alter deutlich an.

- Zwei Drittel aller älteren Menschen nehmen psychotrope - einschließlich pflanzlicher Mittel - ein, ein Viertel konsumiert regelmäßig verschreibungspflichtige Psychopharmaka.

- 19\% der Wachzeit werden mit Ruhephasen verbracht. Diese Ruhephasen nehmen mit zunehmendem Alter zu.

- Hinsichtlich gedanklicher Themen geben 70\% der untersuchten Personen an, sich mit dem Wohlergehen ihrer Angehörigen zu beschäftigen. 6o\% sagten, dass sie sich mit ihrer eigenen geistigen Leistungsfähigkeit beschäftigen - und nur 30\% sagten, dass sie sich stark mit den Themen Sterben und Tod auseinandersetzen.

- Gedächtnisleistungen nehmen mit zunehmendem Alter ab, wobei vor allem Details aktueller Geschehnisse eher vergessen werden als zentralere Themen. Doch bleiben diese Personen bis in das hohe Alter lernfähig.

- 70\% glaubten, ihre Geschicke nach wie vor selbst regeln zu können und 94\% hatten nach wie vor zukunftsorientierte Lebensziele. 40\% glaubten, am meisten über die Gegenwart, 30\% über die Vergangenheit und 25\% über die Zukunft nachzudenken.

- 55\% gaben an, vertraute Personen zu haben, mit denen sie über alles reden können, was bei $45 \%$ nicht der Fall war.

\subsection{Prävention, Beratung und Therapie beim alternden Mann}

Die Andrologie beschäftigt sich schon seit sehr vielen Jahren mit Männerproblemen allerdings schwerpunktmäßig zunächst mit der Zeugungsfähigkeit des Mannes sowie mit sexuellen Problemen. Hinsichtlich eines interdisziplinären Ansatzes jedoch etablieren sich erste Männergesundheitszentren in Deutschland (Goeschel u. Bollmann 2007). Auch gibt es mittlerweile Zentren für Männergesundheitsberatung, deren Zugang kostenfrei und anonym erfolgt, um Männer für ein gesundheitsbewussteres Verhalten und dem Zugang zum etablierten Gesundheitsbetrieb zu motivieren. Beim Frankfurter Männerberatungskonzept (Busch et al. 2004) stehen diesbezüglich präventive, akutkurative, rehabilitative und edukative Aufgaben im Vordergrund.

Auch auf universitärer Ebene gibt es interdisziplinäre Männersprechstunden - so etwa in der eigenen Einrichtung des Leipziger Universitätsklinikums im Rahmen des Europäischen Trainingszentrums für Andrologie, wo Medizin und Psychologie wie Psychotherapie gleichermaßen vertreten sind.

Aber selbst auf kommunaler Ebene gibt es wegen des demografischen Wandels Bemühungen, älter werdende Menschen (Männer und Frauen gleichermaßen) mehr in soziale Infrastrukturen zu integrieren (Dienel u. von Blanckenburg 2010; Klie 2010) Dazu gehört z.B. auch die Förderung und Unterstützung des Aufbaus von Einrichtungen für professionelle Entwicklungsberatung und Männerselbsthilfegruppen mit paraprofessionellen Helfern (Gräser 2007). Auch werden verschiedene Präventionsund Trainingsansätze im höheren Alter diskutiert (Kruse 2007). Interventionen solcher Ansätze sind gerichtet auf Maßnahmen zum Erhalt und auch der Wiedergewinnung von geistigen und körperlichen Fähigkeiten, zur Förderung eines aktiven Lebensstils u.a. 


\section{Literatur}

Albani C, Gunzelmann T, Brähler E (2009) Körperbild und körperliches Wohlbefinden im Alter. Z Gerontol Geriat 42, 236-244

Backes GM, Clemens W (2008) Lebensphase Alter. Eine Einführung in die sozialwissenschaftliche Alternsforschung. Weinheim und München: Juventa, 3. Aufl

Baltes PB, Mittelstraß I, Staudinger UM (Hrsg.) (1994) Alter und Altern: Ein intersidziplinärer Studientext zur Gerontologie. Berlin, New York: Walter de Gruyter

Beutel ME, Glaesmer H, Wiltink I, Marian H, Brähler E (2010) Life satisfaction, anxiety, depression and resilience across the life span of men. The Aging Male 13, 32-39

Brandtstädter I, Lindenberger U (Hrsg.) (2007) Entwicklungspsychologie in der Lebensspanne. Ein Lehrbuch. Stuttgart: Kohlhammer

Busch 0, Eishold M, Jonas D, Bickeböller R (2004) Konzept und Erfahrungen der Frankfurter Männergesundheitsberatung. Blickpunkt Der Mann 2, 10-14

Daig I (2009) Maskulinität und Maskulinitäten. In: Kleiber D, Grüsser S, Knoll N, Brähler E (Hrsg.) Tabuzonen der Frauen- und Männergesundheit. Psychosoziale Perspektiven. Gießen: Psychosozial-Verlag, 63-79

Dienel HL, von Blanckenburg C (2010) Soziale Infrastrukturen und bürgerschaftliches Engagement von und für junge Alte(n). Z Gerontol Geriat 43, 77-81

Dinges M (2009) Männer, die beratungsresistenten Gesundheitsidioten? Blickpunkt Der Mann 7, 19-23

Dinges M (2007a) Was bringt die historische Forschung für die Diskussion zur Männergesundheit? Blickpunkt Der Mann 5, 6-9

Dinges M (2007b) Historische Forschung und die aktuelle Diskussion zur Männergesundheit. In Stiehler M, Klotz T (Hrsg) Männerleben und Gesundheit. Eine interdisziplinäre, multiprofessionelle Einführung. Weinheim und München: Juventa, 24-35

Filipp SH (2007) Kritische Lebensereignisse. In: Brandtstädter J, Lindenberger U (Hrsg.) Entwicklungspsychologie in der Lebensspanne. Ein Lehrbuch. Stuttgart: Kohlhammer, 337-366

Gerok W, Brandstädter I (1994) Normales, krankhaftes und optimales Altern: Variantions- und Modifikationsspielräume. In: Baltes PB, Mittelstraß I, Staudinger UM (Hrsg.) Alter und Altern: Ein Ein intersidziplinärer Studientext zur Gerontologie. Berlin, New York: Walter de Gruyter, 356-385

Glaesmer H, Kallert TW, Brähler E, Hofmeister D, Gunzelmann T (2010) Die Prävalenz depressiver Beschwerden in der älteren Bevölkerung der Bundesrepublik Deutschland und die Bedeutung methodischer Aspekte für die identifizierten Prävalenzen. Psychiat Prax 37, 71-77

Goeschel A, Bollmann M (2007) Zentren für Männergesundheit: Versorgungsform zur Schließung der „Marktlücke“ Männerbehandlung. Blickpunkt Der Mann 5, 31-36

Gräser H (2007) Entwicklungsberatung. In: Brandtstädter J, Lindenberger U (Hrsg.) Entwicklungspsychologie in der Lebensspanne. Ein Lehrbuch. Stuttgart: Kohlhammer, 599-623

Harth W, Hermes B, Jasch K, Seikowski K (2007) Somatoforme Störungen in der Andrologie. Blickpunkt DER MANN, 5, 25-28

Harth W, Seikowski K, Hermes B, Gieler U (2008) Lifestyle_Medikamente in der Medizin. Wien Med Wochenschr 158, 110-115

Klie T (2010) Alter und Kommune. Z Gerontol Geriat 43, 75-76

Kruse A (2007) Präventions- und Trainingsansätze im höheren Alter. In: Brandtstädter I, Lindenberger U (Hrsg.) Entwicklungspsychologie in der Lebensspanne. Ein Lehrbuch. Stuttgart: Kohlhammer, 624-655

Lindenberger U, Smith J, Mayer KU, Baltes PB (Hrsg.) (2010) Die Berliner Altersstudie. Berlin: Akademie-Verlag, 3. Aufl

Merbach M, Beutel M, Brähler E (2007) Gesundheit im Alter. In: Stiehler M, Klotz T (Hrsg.) Männerleben und Gesundheit. Eine interdisziplinäre, multiprofessionelle Einführung. Weinheim, München: Juventa Verlag, 90-103

Richter V, Guthke I (1996) Leipziger Ereignis- und Belastungsinventar (LEBI). Göttingen, Bern, Toronto, Seattle: Hogrefe

Seikowski K (2008) Männer und Gesundheit. In: Berth H, Balck F, Brähler E (Hrsg.) Medizinische Psychologie und Medizinische Soziologie von A bis Z. Göttingen: Hogrefe, 287-289 
III Männerpsyche: Männer ticken anders

Seikowski K, Stöbe K, Harth W (2007) Midlife Crisis bei Männern? - Subjektiv wahrgenommene körperliche und psychische Veränderungen bei Männern zunehmenden Alters. MMW - Fortschritte der Medizin 149, 132-136

Statistisches Bundesamt (2009) Die Bevölkerung bis 2060. 12. koordinierte Bevölkerungsvorausberechnung

Thiele A (1999) Körperliches Altern und psychisches Befinden: Theoretische Überlegungen und empirische Ergebnisse aus einer Studie zum alternden Mann. Z Med Psychol 1, 159-166

Wolfersdorf M (2009) Männersuizid: Warum sich „erfolgreiche“ Männer umbringen - Gedanken zur Psychodynamik. Blickpunkt Der Mann 7, 38-41 